\title{
Description-Based Mashup of Web Applications
}

\author{
Junxia Guo and Takehiro Tokuda \\ Department of Computer Science, Tokyo Institute of Technology \\ Ookayama 2-12-1-W8-71, Meguro, Tokyo 152-8552, Japan \\ \{guo, tokuda\}@tt.cs.titech.ac.jp
}

\begin{abstract}
The World Wide Web has become an almost limitless source of information. Mashup combines information or functionality from two or more existing Web sources to create a new Web page or application. Unfortunately, it is difficult for users without programming experiences to build mashups with existing Web applications, especially when they want to transfer information between Web applications which are used to build mashup. In this paper, we propose a description-based mashup approach for personal use which allows users without programming experiences to build mashup applications with existing Web applications as well as to transfer information between Web applications. This approach is based on information extraction, information transfer and functionality emulation methods. Our implementation shows that general Web applications can be used to build mashup applications easily without programming.
\end{abstract}

Keywords: Web application, description-based mashup, information transfer, information extraction.

\section{Introduction}

With the development of World Wide Web, more and more information has become available on the Web. The Web can be considered as an infinite source of information. In recent years, mashup applications have brought new creativity and functionality to Web applications by combining data or functionality from two or more existing Web sources. The Web sources for building mashup applications can be Web applications and Web services. Although Web services are most commonly used Web sources of mashup, many existing Web sites do not provide Web services, such as the BBC Country Profiles [4]. Thus, it is not easy to build mashup applications with existing Web applications especially for the users without programming experiences.

Methods to help users without programming experiences to build mashups easily and efficiently have been improved rapidly in recent years. Most of the existing mashup methods build mashups only with Web services and do not support the use of Web applications.

Some mashup methods can integrate parts of Web applications without Web services, but they have certain limitations. For example, C3W [2] can reuse text and functional parts of Web pages by clipping, connecting and cloning to build 
mashups. However, C3W is not straightforward to general users because of a special browser to clip Web content from Web pages and a special container named DerivationPad for the clipped Web content.

Florian Daniel et al. proposed an approach in [1] that allows users to compose mashup applications using UI components. But it has a premise that component developers (IT specialists) need to componentize UI components from the Web applications and publish them on the Web.

Dapp Factory [5] can use information extracted from Web pages and RSS to build mashups, but it cannot extract dynamic Web content from Web pages such as the clock part of Localtimes.info [6]. Here we use the term static Web content to represent the content shown on the Web page that can be found directly in the HTML source file, for example text and image. And we use the term dynamic Web content to represent the content that is created by clientside scripts dynamically, which is shown on the Web page but cannot be found directly in the HTML source file.

\section{Problem Statement and Research Objectives}

As we mentioned in Section 1, most of the existing personal-use-oriented mashup tools for users without programming experiences cannot address two problems. One problem is that they cannot use dynamic Web content of Web pages as mashup components. The other problem is that they do not support the information transfer between the mashup components. By transfer here we mean the information extracted from mashup component $\mathrm{A}$ is given to mashup component $\mathrm{B}$ as the input. And in this paper, the mashup component is the component that is generated from existing Web applications.

In this paper, we present an approach that allows users without programming experiences to build mashup applications with any parts of existing Web applications and to transfer information between mashup components. We mainly have two objectives.

- Extract not only static Web content, but also dynamic Web content from Web applications and use the extracted results as elements to build mashups.

- Implement information transfer between mashup components extracted from Web applications without programming.

\section{Research Methodology}

To achieve above objectives, we need to address two main problems. One is how to wrap part(s) of a Web application into a mashup component. The other one is how to transfer information between the mashup components. The technique that we use to wrap part(s) of a Web application into a mashup component is based on information extraction and functionality emulation methods. We record the information of mashup components into a description file, which is written by notation called Mashup Component Description Language file (MCDL file) to 
configure the locations and scopes of target parts of Web applications. To transfer information between mashup components, we also record the information about the data that will be delivered to other components in MCDL file. Then, we obtain the data using information extraction method and transfer the data using the program hidden in the client-side mashup application.

The components that we use to build mashup application are extracted from general Web applications. To describe the necessary information for the extraction, emulation and information transfer, we use Mashup Components Description Language. It is XML-based file, where following items are defined for each mashup component. At present we have to write each item manually. We plan to generate the following description automatically.

- id: We specify the unique name of each mashup component.

- type: We specify the type of the mashup component. It should be one of \{Application, Feed, SOAP and REST . In this paper, we focus on the "Application" type.

- startPageURL: We specify the URL of a Web page where we can submit the searching request to get the target Web page.

- inputType: We specify the type of searching method on the start page. It should be one of \{LinkList (anchor list), OptionList (drop-down option list in selectbox) or InputBox (text input field) and None\}. We just support these four basic input types by now. We plan to do experiments about other input types, for example radio button, as future work.

- keywordFrom: We specify how to get the searching keyword of emulation. It could be the input value from user interface, or the value transferred from other component. When the searching keyword is from other component, we use the "id" of the component to describe it.

- inputArea: We specify the path of the input Web content on the start page. If there is other content with the same "inputType" in the start page, we need to specify which one is going to be used. Users can give a value of "null" instead of the path, which will be treated as the path of body node by default. The path that we use here and other items are XPath-like expressions. The value of path can be gotten easily by using the tool we supply.

- targetContent: We specify the Web content to be extracted by path. Here we support multiple Web contents extraction.

- targetContentType: We specify the data type of the target Web content. It could be dynamic content (mashup, flash or function part) or static content (body, bullet, bullet list, definition list bullet, definition list, div, form, heading, input, label, text, image, link, object, select, paragraph, span, table or text area).

- newStyleforContent: We specify the new style information of target Web content in the mashup resulting page. However, the style can only be changed for static Web contents. According to the type of target Web content, different style attributes can be specified. We process this based on the attribute of different tags defined in HTML. The "newStyleforContent" information is optional information, and when it is "null", extracted Web content will be 
shown in its original style. For dynamic Web contents, the extracted parts are shown in their original styles and the "newStyleforContent" value is "null".

- valueToOtherComponent: We specify the information that will be transferred to other component by path.

The above ten items describe how to get a component. A MCDL file describes a series of components from different Web sources, like a batch file. Each "component" defined in the MCDL file will be shown in one container. In the above ten items, "type", "inputType", "keywordFrom", "targetContentType" and "newStyleforContent" are selected from the predefined list. "startPageURL" is copied from browser. "inputArea", "targetContent" and "valueToOtherComponent" can be copied from our Path-Reader Tool[3], which can generate path strings of target Web content by GUI. Users can get the paths easily by clicking the target parts and then COPY the path into MCDL file. Users do not need to read the HTML source code manually.

As shown in Figure 1, the whole process consists of the following steps.

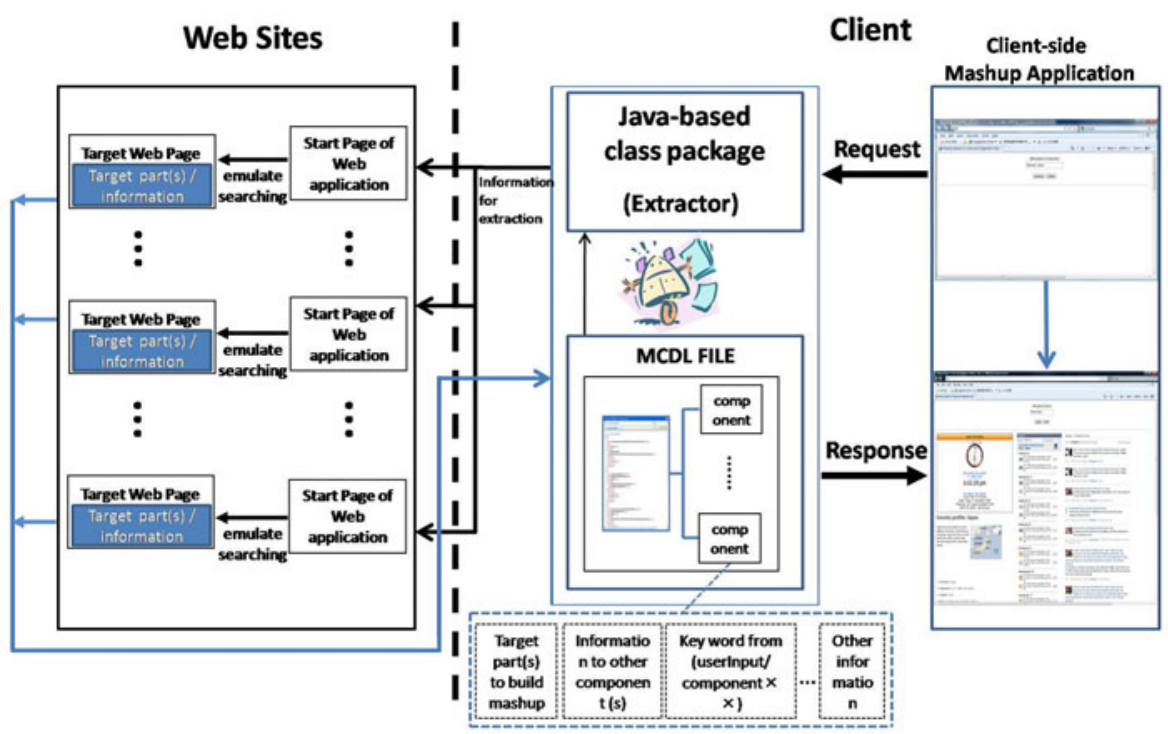

Fig. 1. Overview of Our Approach

Step 1: Users create a MCDL file which includes the information of mashup components that will be used to build mashup application and the information that will be transferred between the components. We provide an application to ease the creation of the MCDL file.

Step 2: Users send requests from the "Client-side Mashup Application" to the "Extractor". 
Step 3: The "Extractor" reads the MCDL file, then according to the given keyword searches and extracts the target parts which are specified in the MCDL file.

Step 4: We integrate the extracted components and arrange the layouts as the response result.

\section{Expected Contributions and Plan for Future Work}

In this paper, we have presented a novel approach that allows users to integrate any parts from any Web applications without programming and allows the information transfer between mashup components to realize the consecutive queries for personal use. Our approach uses a description language (MCDL) to describe the information about mashup components which is selected to be extracted from Web applications and the information about information transfer between mashup components.

In future work, we would like to explore more flexible ways of integrating Web applications and Web services. At present we support one path to describe the location of "targetContent". We would like to support multiple paths to describe the location of "targetContent" and allow the users to decide how many paths they would like to use according to the accuracy they want. Furthermore, we would like to explore an approach to help the users without programming experiences to build mashup applications with SOAP type Web services easily and efficiently without restriction. We also would like to supply a tool to generate the MCDL file easily and automatically. In addition, the information about mashup components and the information about mashup application logic is described in one file at present. It is not convenient for reusing the components. We would like to separate the two aspects.

\section{References}

1. Daniel, F., Matera, M.: Turning Web Applications into Mashup Components: Issues, Models, and Solutions. In: Proceeding of the 9th International Conference on Web Engineering, ICWE 2009 (2009)

2. Fujima, J., Lunzer, A., Hornbak, K., Tanaka, Y.: C3W: Clipping, Connecting and Cloning for the Web. In: Proceeding of the 13th International Conference on World Wide Web (2004)

3. Guo, J., Han, H., Tokuda, T.: A New Partial Information Extraction Method for Personal Mashup Construction. In: Proceeding of the 19th European-Japanese Conference on Information Modelling and Knowledge Bases (2009)

4. BBC Country Profiles, http://news.bbc.co.uk/2/hi/country_profiles/default.stm

5. Dapp Factory, http://www. dapper.net/

6. Localtimes.info, http://localtimes.info/ 